\title{
Design Analysis of a Liquid-Piston Compression Chamber with Application to Compressed Air Energy Storage
}

\author{
Chao Zhang*, Perry Y. Li*, James D. Van de Ven*, Terrence W. Simon* \\ * Mechanical Engineering Department, University of Minnesota, 111 Church St. S.E. 55455, Tel: +1 612625 5831; \\ fax: +1 612624 5230, E-mail address: simon002@umn.edu (T.W. Simon).
}

\begin{abstract}
The present study focuses on a design analysis of a shaped liquid piston compression chamber based on CFD. The liquid piston compression chamber is for application to Compressed Air Energy Storage (CAES), which can be used to even the mismatch between power generation and power demand, and, thus, the objective of the design exploration is to maximize the compression efficiency. Within the compression chamber is an open-cell metal foam medium for enhancement of heat transfer. Traditionally, the chamber has a cylindrical shape. The present study explores the effects on compression efficiency of varying the profile of cross-sectional diameter along the axis of the chamber. This leads to a compression chamber with curved walls that assumes a gourd-like shape. A set of exploratory design cases is completed using the orthogonal array concept based on the Taguchi method, hence reducing the number of realizations. CFD simulations provide insight into how the chamber shape affects the flow physics during compression. A quantitative design analysis shows that, in general, a large aspect ratio and a steep radius change of the chamber is preferred, which is in line with a visualization of the CFD flow fields. The relative importance of each different shape parameter is analyzed.
\end{abstract}

Keywords: Heat Transfer, Liquid Piston, CFD, Compressed Air Energy Storage.

\section{INTRODUCTION}

A design analysis for a shaped liquid piston compression chamber for application to Compressed Air Energy Storage (CAES) is presented. The CAES stores energy (e.g. from wind turbines) by compressing air during low power demand periods, liberating it by expanding compressed air during high power demand periods [1]. The benefit is that it evens the mismatch between power generation and power demand, such as experienced with wind generation. Analyses based on CFD simulations have shown that compression efficiency is higher when the compression process is more isothermal, for the same pressure compression ratio [2]. Typically, compression results in a temperature rise of the compressed air. This temperature rise occurs as a result of part of the compression work being converted into an increase in internal energy of the air. This internal energy rise is wasted, however, during the storage period as the compressed air cools to ambient temperature. Therefore, cooling the air during compression is important for lowering input work and maintaining high compression efficiency. Avoiding high temperatures is important also for materials and durability reasons.

A liquid piston approach can be used for the CAES compression. The sealing effectiveness of a liquid piston gives an advantage over a traditional solid piston in terms of power consumption [3]. A more important advantage of the liquid piston to CAES is that it offers the possibility of inserting porous media into the chamber to enhance heat transfer, as liquid can flow through the pores of porous medium. Detailed modeling and CFD simulations have been conducted on liquid piston compressors with open-cell metal foam inserts for application to lower pressure compression ratios. Although the porous medium introduces resistance to the flow, which causes pressure drop, the power loss due to this is minuscule relative to the power needed to compress the air. The results show that such compressors benefit from the cooling effect afforded by a liquid piston and the accompanying heat absorbing media for improving compression efficiency [4].

Numerical modeling of the liquid piston compressor with the inserts of porous media is done with volume-averaging techniques $[5,6]$. The continuity, momentum and energy equations are volume-averaged on the scale of a Representative Elementary Volume (REV) of the porous medium. Instead of resolving the flow through the exact shape of the porous medium, these volume-averaged equations are solved. As a result, flow through a porous medium is solved in a continuum domain without the need for resolving the pore-scale activities directly; a negative momentum source term arises in the momentum equation that represents the pore-scale activities and satisfies closure. This effect is often modeled by Darcian and inertial terms [7]. Two volume-averaged energy equations, one each for the solid and fluid, are solved, coupled by an interfacial heat transfer term. Since the liquid piston chamber invoves two phases, water and air, the Volume Of Fluid (VOF) method [8] is used in conjunction with the porous media model.

Traditionally, the compression chamber has a cylindrical shape. The present study explores the compression efficiency change that is derived by varying profiles of cross-sectional diameter along the axis of the chamber. This leads to a compression chamber with curved walls that make it assume a gourd-like shape. The advantage of such shape is that it offers an opportunity for more complex flow features in the chamber during compression that enhance mixing and heat transfer. To 
analyze the wall shape effect, the shape is parameterized and a series of design exploration cases based on orthogonal arrays is created. The orthogonal arrays of experiments have been often used with the Taguchi optimization method [9], and shown to be capable of reducing the number of realizations, whether experimental or numerical $[10,11]$. In the present study, four shape parameters are recognized, each having four different values of parameters of interest. Based on the orthogonal array method, sixteen orthogonal arrays of the design are created, each is analyzed by a CFD run. The results give insight into the fluid flow and heat transfer characteristics in the compression chamber and lead to an optimized chamber shape within the chosen parameter domain.

\section{ORTHOGONAL ARRAYS OF EXPLORATORY DESIGNS}

The present study investigates the effects of the shape of a liquid piston compression chamber. A schematic of a shaped chamber is shown in Fig. 1. Within the chamber is inserted an open-cell metal foam porous medium of 10 Pores Per Inch (PPI) and 93\% porosity. As water is pumped into the chamber (on the left of the figure - note that gravity is from right to left in this figure), a rising water-air interface acts as a piston surface that compresses the air in the chamber. The compression process starts with pre-compressed air at 7 bars and $293 \mathrm{~K}$ and ends at 210 bars after 3 seconds. The chamber volume is fixed at $2.19 \times 10^{-3} \mathrm{~m}^{3}$ in this study. The chamber's main shape is governed by four parameters, as labelled in Fig. 1: the length, the inlet radius, the maximum radius, the top cap radius and the location of the maximum radius with respect to the inlet.

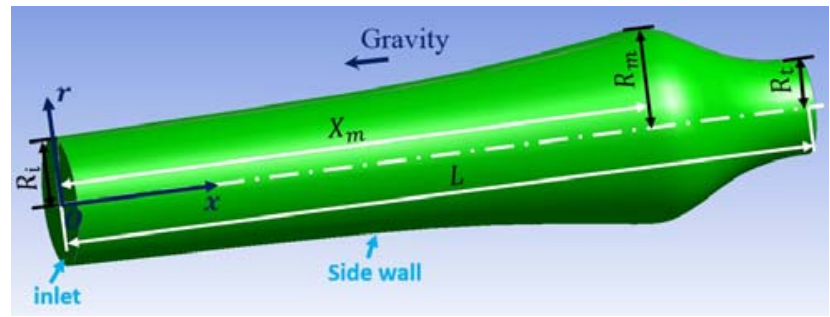

Fig. 1. Schematic of liquid piston chamber and shape parameters

Of interest to the design is analysis with the different ratios of these five shape parameters, since the chamber volume is fixed. Four dimensionless shape parameters based on these five length scales are created:

$$
\begin{aligned}
& \mathcal{P}_{1}=\frac{L}{R_{m}} \\
& \mathcal{P}_{2}=\frac{R_{m}}{R_{i}} \\
& \mathcal{P}_{3}=\frac{X_{m}}{L} \\
& \mathcal{P}_{4}=\frac{R_{m}}{R_{t}}
\end{aligned}
$$

The domains of interest for these parameters are: $1 \leq \mathcal{P}_{1} \leq 8$, $1.5 \leq \mathcal{P}_{2} \leq 6,0.333 \leq \mathcal{P}_{3} \leq 0.8,1.5 \leq \mathcal{P}_{4} \leq 6$. Four levels (values) for each parameter within its own domain are chosen for investigation. They are given in Table 1. Using the "L'16
Array," based on the Taguchi design method [9], sixteen orthogonal exploratory shape designs are created, and given in Table 2. A design is an array of shape parameter values, and it is orthogonal to other arrays of parameter values. The orthogonal array method can be proven mathematically. A practical perspective is that (1) each design is most different from the others, and (2) in all 16 designs, each level of each parameter has been combined with all other levels of other parameters. CFD simulations are done for different designs.

Table 1. Levels of investigation for different parameters

\begin{tabular}{|l|l|l|l|l|}
\hline & $\mathcal{L}_{1}$ & $\mathcal{L}_{2}$ & $\mathcal{L}_{3}$ & $\mathcal{L}_{4}$ \\
\hline $\mathcal{P}_{1}$ & 1 & 2 & 4 & 8 \\
\hline $\mathcal{P}_{2}$ & 1.5 & 2 & 4 & 6 \\
\hline $\mathcal{P}_{3}$ & 0.333 & 0.5 & 0.65 & 0.8 \\
\hline $\mathcal{P}_{4}$ & 1.5 & 2 & 4 & 6 \\
\hline
\end{tabular}

Table 2. Exploratory designs based on orthogonal arrays

\begin{tabular}{|l|r|r|r|r|}
\hline & \multicolumn{1}{|c|}{$\mathcal{P}_{1}$} & \multicolumn{1}{|c|}{$\mathcal{P}_{2}$} & \multicolumn{1}{|c|}{$\mathcal{P}_{3}$} & \multicolumn{1}{c|}{$\mathcal{P}_{4}$} \\
\hline Design 1 & $1\left(\mathcal{L}_{1}\right)$ & $1.5\left(\mathcal{L}_{1}\right)$ & $0.333\left(\mathcal{L}_{1}\right)$ & $1.5\left(\mathcal{L}_{1}\right)$ \\
\hline Design 2 & $1\left(\mathcal{L}_{1}\right)$ & $2\left(\mathcal{L}_{2}\right)$ & $0.5\left(\mathcal{L}_{2}\right)$ & $2\left(\mathcal{L}_{2}\right)$ \\
\hline Design 3 & $1\left(\mathcal{L}_{1}\right)$ & $4\left(\mathcal{L}_{3}\right)$ & $0.65\left(\mathcal{L}_{3}\right)$ & $4\left(\mathcal{L}_{3}\right)$ \\
\hline Design 4 & $1\left(\mathcal{L}_{1}\right)$ & $6\left(\mathcal{L}_{4}\right)$ & $0.8\left(\mathcal{L}_{4}\right)$ & $6\left(\mathcal{L}_{4}\right)$ \\
\hline Design 5 & $2\left(\mathcal{L}_{2}\right)$ & $1.5\left(\mathcal{L}_{1}\right)$ & $0.5\left(\mathcal{L}_{2}\right)$ & $4\left(\mathcal{L}_{3}\right)$ \\
\hline Design 6 & $2\left(\mathcal{L}_{2}\right)$ & $2\left(\mathcal{L}_{2}\right)$ & $0.333\left(\mathcal{L}_{1}\right)$ & $6\left(\mathcal{L}_{4}\right)$ \\
\hline Design 7 & $2\left(\mathcal{L}_{2}\right)$ & $4\left(\mathcal{L}_{3}\right)$ & $0.8\left(\mathcal{L}_{4}\right)$ & $1.5\left(\mathcal{L}_{1}\right)$ \\
\hline Design 8 & $2\left(\mathcal{L}_{2}\right)$ & $6\left(\mathcal{L}_{4}\right)$ & $0.65\left(\mathcal{L}_{3}\right)$ & $2\left(\mathcal{L}_{2}\right)$ \\
\hline Design 9 & $4\left(\mathcal{L}_{3}\right)$ & $1.5\left(\mathcal{L}_{1}\right)$ & $0.65\left(\mathcal{L}_{3}\right)$ & $6\left(\mathcal{L}_{4}\right)$ \\
\hline Design 10 & $4\left(\mathcal{L}_{3}\right)$ & $2\left(\mathcal{L}_{2}\right)$ & $0.8\left(\mathcal{L}_{4}\right)$ & $4\left(\mathcal{L}_{3}\right)$ \\
\hline Design 11 & $4\left(\mathcal{L}_{3}\right)$ & $4\left(\mathcal{L}_{3}\right)$ & $0.333\left(\mathcal{L}_{1}\right)$ & $2\left(\mathcal{L}_{2}\right)$ \\
\hline Design 12 & $4\left(\mathcal{L}_{3}\right)$ & $6\left(\mathcal{L}_{4}\right)$ & $0.5\left(\mathcal{L}_{2}\right)$ & $1.5\left(\mathcal{L}_{1}\right)$ \\
\hline Design 13 & $8\left(\mathcal{L}_{4}\right)$ & $1.5\left(\mathcal{L}_{1}\right)$ & $0.8\left(\mathcal{L}_{4}\right)$ & $2\left(\mathcal{L}_{2}\right)$ \\
\hline Design 14 & $8\left(\mathcal{L}_{4}\right)$ & $2\left(\mathcal{L}_{2}\right)$ & $0.65\left(\mathcal{L}_{3}\right)$ & $1.5\left(\mathcal{L}_{1}\right)$ \\
\hline Design 15 & $8\left(\mathcal{L}_{4}\right)$ & $4\left(\mathcal{L}_{3}\right)$ & $0.5\left(\mathcal{L}_{2}\right)$ & $6\left(\mathcal{L}_{4}\right)$ \\
\hline Design 16 & $8\left(\mathcal{L}_{4}\right)$ & $6\left(\mathcal{L}_{4}\right)$ & $0.333\left(\mathcal{L}_{1}\right)$ & $4\left(\mathcal{L}_{3}\right)$ \\
\hline
\end{tabular}

\section{CFD MODELING}

Each of the designs in Table 2 is analyzed by CFD. The compression chamber is studied in cylindrical coordinates. As shown in Fig. 1, the $x$ axis is along the centerline of the compression chamber. The gravitational field points opposite to the axis direction $x$. At time $=0$, water begins to be pumped at a constant rate into the chamber. During compression, water, air, and solid coexist in the chamber.

The CFD simulations utilize a technique that combines a two-energy-equation porous media model (solid and fluid) and a Volume of Fluid (VOF) analysis method for two-phase flow simulation. The continuity equation for each of the two fluid phases is solved. The transport of the two fluid phases is numerically represented by the scalar functions of phase volume fraction, $\alpha$. Let subscripts 1 and 2 represent air and water, respectively. Then, the continuity equations are:

$$
\begin{aligned}
& \frac{\partial \alpha_{1} \rho_{1}}{\partial t}+\nabla \cdot\left(\alpha_{1} \rho_{1} \vec{u}\right)=0 \\
& \frac{\partial \alpha_{2}}{\partial t}+\nabla \cdot\left(\alpha_{2} \vec{u}\right)=0
\end{aligned}
$$


One set of momentum and energy equations is solved for the mutiphase flow mixture, based on averaged properties:

$$
\begin{gathered}
\frac{\partial \rho \vec{u}}{\partial t}+\nabla \cdot(\rho \vec{u} \vec{u})=-\nabla p+\nabla \cdot \overline{\bar{\tau}}+\rho \vec{g}+\vec{S}_{m} \\
\frac{\partial \epsilon\left(\rho c_{p} T\right)}{\partial t}+\epsilon \nabla \cdot\left(\vec{u} \rho c_{p} T\right)=\epsilon \nabla \cdot k_{f} \nabla T+h_{V}\left(T_{s}-T\right)+\epsilon \frac{\partial p}{\partial t}
\end{gathered}
$$

where:

$$
\begin{aligned}
& \rho=\alpha_{1} \rho_{1}+\alpha_{2} \rho_{2} \\
& \mu=\alpha_{1} \mu_{1}+\alpha_{2} \mu_{2} \\
& \rho c_{p}=\alpha_{1} \rho_{1} c_{p, 1}+\alpha_{2} \rho_{2} c_{p, 2} \\
& k_{f}=\alpha_{1} k_{1}+\alpha_{2} k_{2}
\end{aligned}
$$

The energy equation for the solid is

$$
(1-\epsilon) \frac{\partial}{\partial t}\left(\rho_{s} c_{s} T_{s}\right)=(1-\epsilon) \nabla \cdot k_{s} \nabla T_{s}-h_{V}\left(T_{s}-T\right)
$$

The momentum sink term, $\vec{S}_{m}$, arises from volumeaveraging the momentum equation on the pore scale of the porous medium. It represents the resistance of the medium to flow. It can be modeled based on a Darcian and an inertial term:

$$
\vec{S}_{m}=-\frac{\mu \epsilon \vec{u}}{K}-\frac{1}{2} b \rho|\epsilon \vec{u}| \epsilon \vec{u}
$$

Previous modeling and experimental studies with the 10PPI metal foam reveals the following resistive properties [4]:

$$
K=2.397 \times 10^{-7} \mathrm{~m}^{2}, \quad b=570.1 / \mathrm{m}
$$

The fluid and solid energy transport equations must be coupled through an interfacial heat transfer term. Previous studies [4] show the following heat transfer correlation can represent the 10PPI metal foam:

$$
\frac{h_{V} d_{m}^{2}}{k}=0.996\left(\frac{\rho \epsilon u d_{m}}{\mu} \operatorname{Pr}\right)^{0.791}
$$

where the mean pore diameter for the 10PPI metal foam is: $d_{m}=3.61 \mathrm{~mm}$. Other physical properties of the analysis are given in Table 3.

Computed are compression processes from $700 \mathrm{kPa}$ to $21 \mathrm{MPa}$. Due to the high pressure, a cubic equation of state for air treated as a real gas is used to relate air pressure to density and temperature [12]:

$$
p=\frac{\rho \Re T}{M-\rho \mathrm{b}}-\frac{\mathfrak{a} \rho^{2}}{M^{2}+\mathrm{b} M \rho}
$$

where,

$$
\begin{aligned}
& \mathfrak{b}=\frac{0.08664 \Re \mathrm{T}_{C}}{P_{c}} \\
& \mathfrak{a}=\frac{0.42727 \Re^{2} T_{c}^{2}}{P_{c}}\left\{1+\mathfrak{n}\left[1-\left(\frac{T}{T_{c}}\right)^{0.5}\right]\right\}^{2} \\
& \mathfrak{n}=0.48+1.574 \omega-0.176 \omega^{2}
\end{aligned}
$$

The acentric factor, $\omega$, is taken to be 0.033 . For air, the critical pressure, critical temperature and molecular weight are respectively, $3.758 \mathrm{MPa}, 132.3 \mathrm{~K}$ and $29 \mathrm{~g} / \mathrm{mol}$. The universal gas constant is $8.314 \mathrm{~J} /(\mathrm{mol} \mathrm{K})$. The specific heat for a real gas is modeled using a departure specific heat $[12,13]$ :

$$
c_{p, 1}=c_{p, 1, \mathrm{ideal}}-c_{p, 1, \mathrm{dep}}
$$

where the $c_{p, 1 \text {,ideal }}$ is for an ideal gas. For air, $c_{p, 1, \text { ideal }}$ is taken as a function of temperature based on a curve fit of the data from [14]. The departure specific heat is based on the following equation,

$$
c_{p, 1, d e p}=-\frac{p}{\rho T}+\frac{\Re}{M}-\frac{\frac{\partial \mathfrak{a}}{\partial T}-\frac{\mathfrak{a}}{T}}{M \mathfrak{b}} \ln \left(1+\frac{\mathrm{b} \rho}{M}\right)
$$

Table 3.Physical Properties [14]

$$
\begin{array}{ll}
\rho_{s}=2719 \mathrm{~kg} / \mathrm{m}^{3} & \rho_{2}=1000 \mathrm{~kg} / \mathrm{m}^{3} \\
k_{s}=205 \mathrm{~W} /(\mathrm{mK}) & c_{2}=4181.3 \mathrm{~J} /(\mathrm{kgK}) \\
c_{s}=871 \mathrm{~J} /(\mathrm{kgK}) & k_{2}=0.56 \mathrm{~W} /(\mathrm{mK}) \\
\mu_{2}=1.002 \times 10^{-3} \mathrm{~Pa} \cdot \mathrm{s} & \\
\mu_{1}=1.716 \times 10^{-5} \times\left(\frac{\mathrm{T}}{273 K}\right)^{2 / 3} \mathrm{~Pa} \cdot \mathrm{s} & \\
k_{1}=\left(0.00468506+\frac{7.16557 \times 10^{-5} \mathrm{~T}}{K}\right) & W /(\mathrm{mK})
\end{array}
$$

CFD simulations are done using finite-volume-based commercial CFD Software ANSYS Fluent. Its default VOF model is used to solve Eqns. (6-9); the solid energy equation, Eq.(14), is implemented through a user-defined scalar equation. The $1^{\text {st }}$ order implicit method is used on the transient discretization. Spatial derivatives are differenced using a $2^{\text {nd }}$ order upwind scheme. The Pressure Implicit with Splitting Operators [15] algorithm is used for pressure-velocity coupling. Also used was The Pressure Staggering Option scheme [16] for calculating discretized pressure values on a staggered grid. The Green-Gauss Cell-Based method is used to calculate the face value of a variable based on the arithmetic average of the values at its adjacent cell centers. In each time step, convergence is satisfied when the residual is smaller than $10^{-9}$. For the 16 runs, the computational grids vary from 60,790 cells to 78,794 cells, all having progresssively fine grids near the wall. The time step sizes of all simulations vary from $1 \times 10^{-5} s$ to $2.5 \times 10^{-5} s$. (The liquid piston speeds vary from $0.049 \mathrm{~m} / \mathrm{s}$ to $1.87 \mathrm{~m} / \mathrm{s}$.) The choice of grid and time step size are in line with previous studies on grid independence criteria [17].

\section{RESULTS}

\subsection{Flow Field}

The temperature distributions at the end of the compression processes for different chamber designs are shown in Figs. 2 17. In these plots, the bottom boundary is the centreline of the chamber and gravity points horizontally from right to left. Water enters the chamber from the left boundary as shown in the figures. The water rises in temperature only very slightly during compression and is occupying regions colored by dark blue, as shown in these plots. Large temperature gradients are present in the air phase. The different chamber shapes affect air temperature distributions differently.

The air temperature distribution in the radial direction at the end of compression is mostly affected by how abruptly the 
chamber's radius changes near the top cap, or the parameter $\mathcal{P}_{4}$. Some of the designs with such abrupt radius changes are shown in in Figs. $2-5$. As can be seen in these plots, when the wall slope is large near the top cap, flow near the side wall next to the water-air interface has a lower temperature due to a radial velocity component, and heat transfer is thus enhanced.

Large air temperature gradients in the axial direction commonly exist in all the chambers. The reason is that flow near the top cap of the chamber is generally in stagnation, which limits the amount of heat transfer between the metal foam and the top cap, and flow near the water-air interface has a high velocity and, thus, offers good heat transfer. However, when the chamber is longer, or has a larger aspect ratio, $\mathcal{P}_{1}$, a relatively smaller percentage of the air volume is in stagnation next to the top cap and a larger portion of the air volume continues to have good heat transfer to the metal foam insert, as shown by Figs. $4-7$.

Although the chamber's shape has complicated effects on the flow and temperature fields, these preliminary observations on the flow distributions show that, in general, large aspect ratios $\left(\frac{L}{R_{m}}\right)$ and abrupt changes in radius may agitate the flow, which is beneficial for heat transfer and cooling of the compressed air.

\subsection{Compression Efficiency}

The thermodynamics efficiencies of different chamber designs are calculated based on CFD results. Compression stores energy in the form of compressed gas for later work extraction. In CAES, the compression efficiency is defined based on the ideal amount of energy extraction, or the storage energy, devided by the actual compression work input $[17,18]$. The storage energy is the amount of work output derived as the compressed air undergoes an isothermal expansion process. It is given by:

$$
E_{s}=P_{0} V_{0}\left[\ln (\zeta)-1+\frac{1}{\zeta}\right]
$$

The total work input is of two parts, the compression work and the cooling work. The compression work is done during compression from $t=0$ to $t=t_{f}$ and is given by

$$
W_{\text {comp }}=\int_{0}^{t_{f}}\left(P_{\text {inlet }}-P_{0}\right) U_{\text {in }} A \mathrm{dt}
$$

After compression, the compressed air cools to the initial temperature through an isobaric process. As the air cools, its volume decreases; thus, additional cooling work is done to maintain pressure:

$$
W_{\text {cool }}=\left(P_{f}-P_{0}\right)\left(V_{f}-V_{0} \frac{P_{0}}{P_{f}}\right)
$$

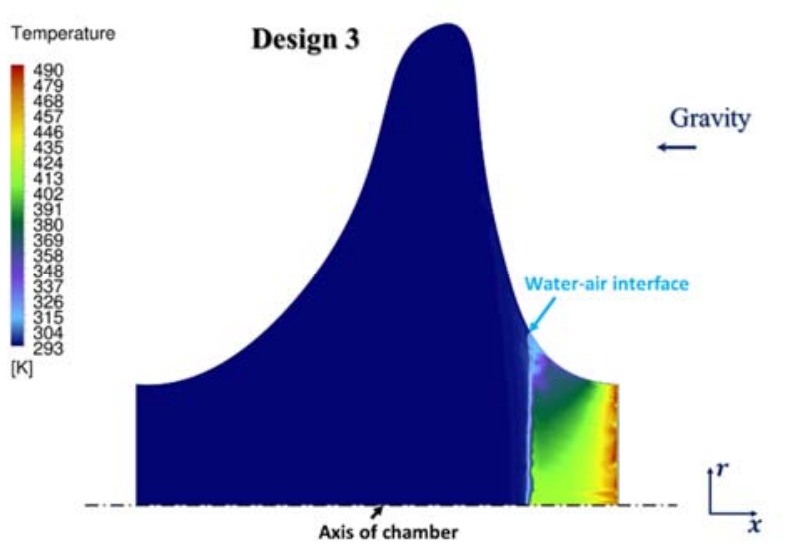

Fig. 2. Temperature field at the end of compression - Design 3

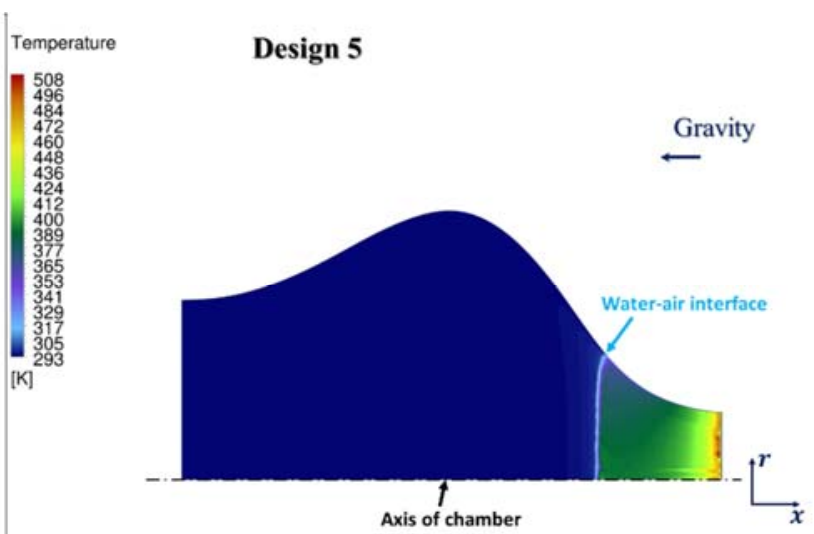

Fig. 3. Temperature field at the end of compression - Design 5

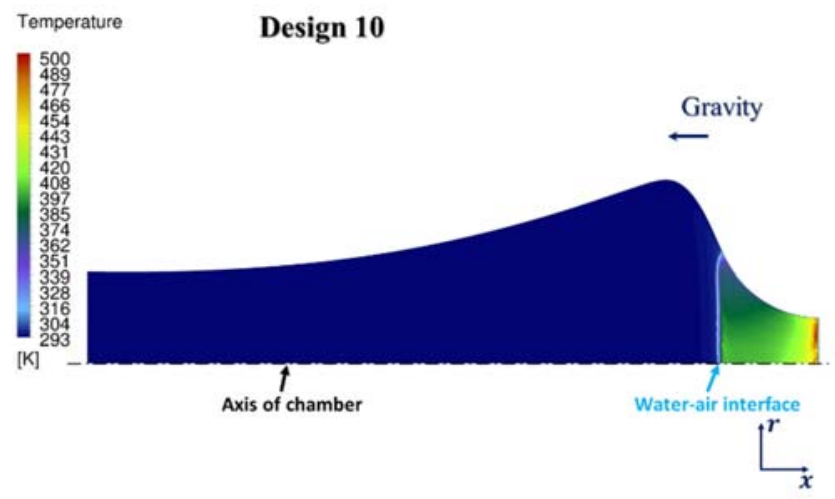

Fig. 4. Temperature field at the end of compression - Design 9

Fig. 5. Temperature field at the end of compression - Design 10 

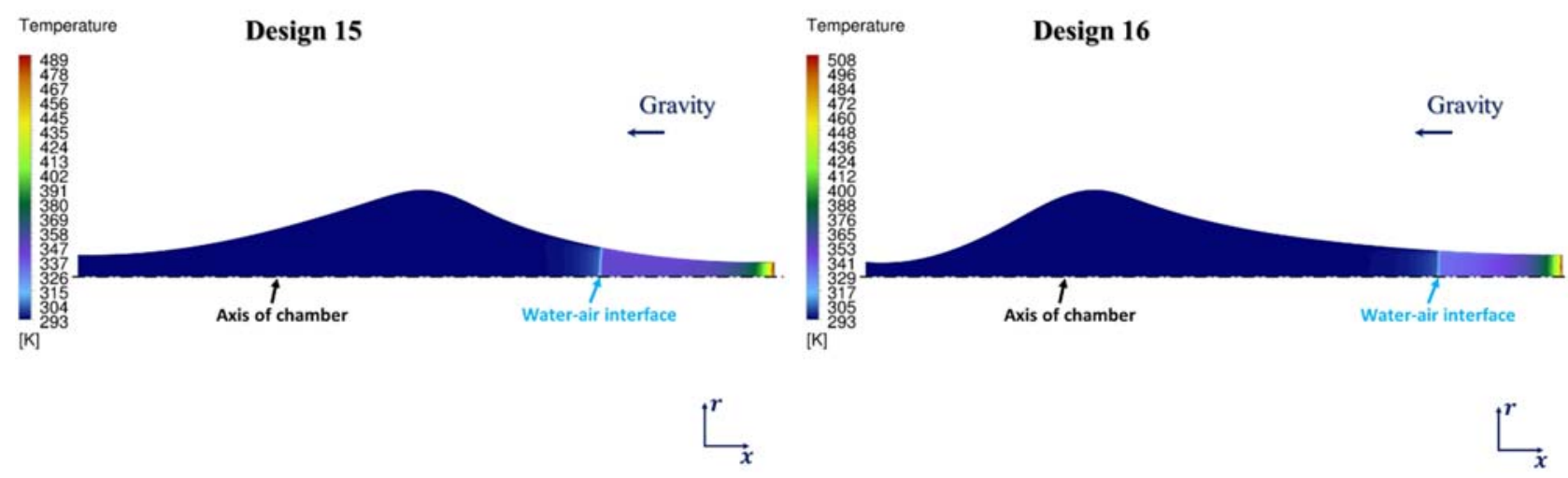

Fig. 6. Temperature field at the end of compression - Design 15

Fig. 7. Temperature field at the end of compression - Design 16

The efficiency of a compression process is the ratio of the storage energy to the total work input,

$$
\eta=\frac{E_{S}}{W_{\text {cool }}+W_{\text {comp }}}
$$

The efficiency values for different chamber designs are calculated and shown in Fig. 8. A comparison of efficiencies among different chambers is in line with the observations made in the previous section. Keeping the chamber longer and making the cross-sectional radius changes steeper is beneficial for achieving higher efficiency (Designs 16 and 15). In general, the steep change of cross-sectional radius results in more radial flow, which enhances mixing; the long, thin shape on the top increases the length that the flow travels, and its speed increases, which offers more opportunity for heat transfer.

Note that in this definition of efficiency, the work that ejects the compressed air from the compression chamber into the storage vessel is not counted with the storage energy. However, the CAES system may be designed such that this ejection work can be used for the next compression cycle, which will result in an increase of about $20 \%$ in efficiency, but the comparison among different chambers remains the same.

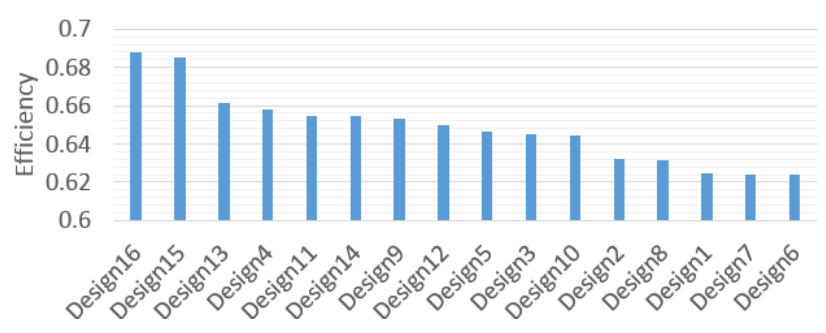

Fig. 8. Efficiency of different chamber designs

\subsection{Design Exploration}

The individual effect of each parameter at each level is calculated. Table 2 shows the sixteen orthogonal arrays with each level of each parameter appearing in four designs. Therefore, the effect of a parameter at a certain level is the average of the efficiency values of the corresponding four designs in which this level of the parameter appears. For example, the effect of $\mathcal{P}_{1}$ at $\mathcal{L}_{1}$ is:

$$
\eta_{\mathcal{P}_{1-} \mathcal{L}_{1}}=\frac{1}{4}\left(\eta_{\text {Design } 1}+\eta_{\text {Design } 2}+\eta_{\text {Design } 3}+\eta_{\text {Design } 4}\right)
$$

Similarly, the effects of all other parameters at different levels are calculated. The results are shown in Fig. 9. For each parameter, the relation between its level and the efficiency is non-linear. For parameters $\mathcal{P}_{1}$ and $\mathcal{P}_{2}$, the minimum efficiencies correspond to their middle levels, in both cases, a parameter value of 2 , and the maximum efficiencies correspond to the maximum levels within their own parameter domains. For parameters $\mathcal{P}_{3}$ and $\mathcal{P}_{4}$, the maximum efficiencies correspond to their middle levels, $\mathcal{L}_{2}$ for $\mathcal{P}_{3}$, and $\mathcal{L}_{3}$ for $\mathcal{P}_{4}$. Therefore, the best design of the chamber would have the following shape parameters:

$$
\mathcal{P}_{1}=8, \quad \mathcal{P}_{2}=6, \quad \mathcal{P}_{3}=0.5, \quad \mathcal{P}_{4}=4
$$
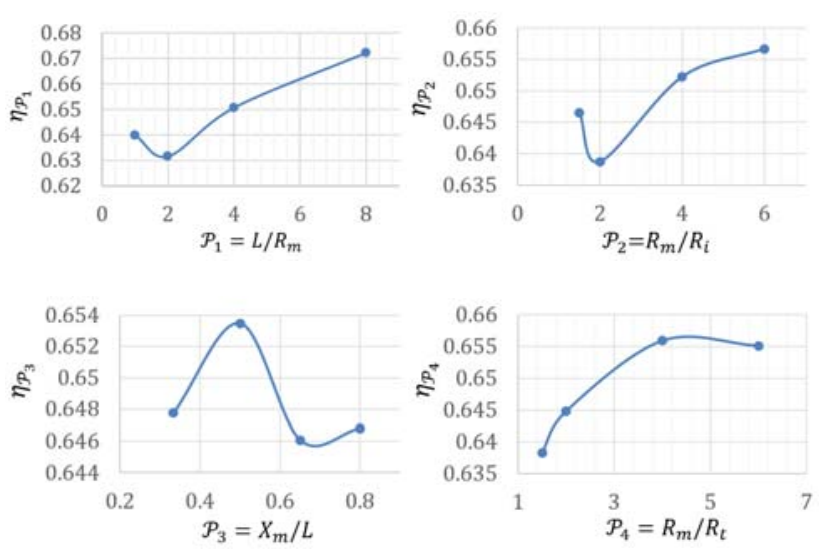

Fig. 9. Individual effects of shape parameters on efficiency

In designing the chamber, changing one shape parameter may not be as effective toward improving the efficiency as changing another. The effects of different parameters are thus compared. For each parameter, a relative effect is calculated by taking the difference between maximum and minimum efficiencies and divide it by the difference between the maximum and minimum levels. The calculated relative effects: $\frac{\Delta \eta}{\Delta \mathcal{P}_{1}}, \frac{\Delta \eta}{\Delta \mathcal{P}_{2}}, \frac{\Delta \eta}{\Delta \mathcal{P}_{3}}$, and $\frac{\Delta \eta}{\Delta \mathcal{P}_{4}}$, are, respectively: 0.0058, 0.0040, 0.0016, and 0.0039 . As can be seen, the aspect ratio of the chamber (the length to the maximum radius ratio) is the most important parameter; the effects of the maximum radius to the inlet radius and to the top radius are similar and less important than the aspect ratio; the axial location of the maximum radius is the least important of the four shape parameters. 


\section{CONCLUSIONS}

A CFD-based design analysis has been done for a liquid piston compression chamber. CFD results reveal detailed flow features in the chamber. When the chamber is longer and has a steeper change in cross-sectional radius, the flow is more agitated and, thus, better heat transfer results. The physics shown by CFD visualization of the flow fields are in line with the design analysis results. A best design has a large aspect ratio and a steep radius change. It is proposed. Relative importance levels of different shape parameters are calculated, which provides further insight into the design.

\section{ACKNOWLEDGMENT}

This work is supported by the National Science Foundation under grant NSF-EFRI \#1038294, and University of Minnesota, Institute for Renewable Energy and Environment (IREE) under grant: RS-0027-11. The authors would like to thank also the Minnesota Super-Computing Institute for the computational resources used in this work.

\section{REFERENCES}

[1] P. Sullivan, W. Short e N. Blari, "Modeling the Benefits of Storage Technologies to Wind Power," in AWEA Wind Power 2008 Conference, Houston, TX, June 2008.

[2] C. Zhang, T. W. Simon e P. Y. Li, "Storage Power and Efficiency Analysis Based on CFD for Air Compressors Used for Compressed Air Energy Storage," in International Mechanical Engineering Congress and Exposition, Houston, TX, Nov. 2012.

[3] J. D. Van de Ven e P. Y. Li, "Liquid Pistong Gas Compression," Applied Energy, vol. 86, n. 10, pp. 21832191, 2009.

[4] C. Zhang, B. Yan, J. Wieberdink, P. Y. Li, J. D. Van de Ven, E. Loth e T. W. Simon, "Thermal analysis of a compressor for application to Compressed Air Energy Storage," Applied Thermal Engineering, vol. 73, n. 2, pp. 1402-1411, 2014

[5] W. G. Gray e K. O'Neill, "On the General Equations for Flow in Porous Media and Their Reduction to Darcy's Law," Water Resources Research, vol. 12, n. 2, pp. 148154, 1976.

[6] C. T. Hsu, "Dynamic Modeling of Convective Heat Transfer in Porous Media," in Handbook of Porous Media (2nd Edition), Taylor and Fracis Group, 2000, pp. 39-80.

[7] K. Vafai e C. L. Tien, "Boundary and Inertial Effects on Flow and Heat Transfer in Porous Media," International Journal of Heat and Mass Transfer, vol. 24, pp. 195-203, 1981.

[8] W. Hirt e B. D. Nichols, "Volume of Fluid (VOF) Method for Dynamics of Free Boundaries," Journal of Computational Physics, vol. 39, pp. 201-225, 1981.

[9] G. Taguchi, T. Yokoyama e Y. Wu, "Taguchi Methods: Design of Experiments," in Quality Engineering Series, Vol. 4, Tokyo, ASI Press, 1993.
[10] W. Xia, H. Y. Hsu e L. X. Kong, "A CFD-Aided Experimental Study on Bending of Micro Glass Pipettes," Journal of Mechanical Science and Technology, vol. 21, pp. 1338-1343, 2007.

[11] Q. Wang, Q. Chen e M. Zeng, "A CFD-Taguchi Combined Method for Numerical Investigation of Natural Convection Cooling Performance of Air-Core Reactor with Noise Reducing Cover," Numerical Heat Transfer, Part A, vol. 55, pp. 1116-1130, 2009.

[12] G. Soave, "Equilibrium Constant from a Modified Redlich-Kwong Equation of State," Chemical Engineering Science, vol. 27, pp. 1197-1203, 1972.

[13] R. H. Aunger, "A Fast, Accurate Real Gas Equation of State for Fluid Dynamics Analysis Applications," Journal of FLuids Engineering, vol. 117, pp. 277-281, 1995.

[14] "Air Properties," The Engineering ToolBox, [Online]. Available: http://www.engineeringtoolbox.com/airproperties-d_156.html. [Consultato il giorno Sep 2014].

[15] R. I. Issa, "Solution of implicitly discretized fluid flow equations by operator-splitting," Journal of Computational Physics, vol. 62, pp. 40-65, 1986.

[16] S. V. Patankar, Numerical Heat Transfer and Fluid Flow, Washington, DC: Hemisphere, 1980.

[17] C. Zhang, J. H. Wieberdink, F. A. Shirazi, B. Yan, T. W. Simon e P. Y. Li, "Numerical Investigation of MetalFoam Filled Liquid Piston Compressor Using a TwoEnergy Equation Formulation Based on Experimentally Validated Models," in ASME 2013 International Mechanical Engineering Congress and Exposition, San Diego, CA, Nov. 2013.

[18] B. Yan, J. H. Wieberding, F. A. Shirazi, B. Yan, P. Y. Li, T. W. Simon, J. D. Van de Ven, "Experimental Study of Heat Transfer Enhancement in a Liquid Piston Copressor/Expander Using Porous Media Inserts," Applied Energy, Vol. 154, n. 15, pp. 40-50

\section{NOMENCLATURE}

$\begin{array}{cll}A & \text { Cross sectional area at the inlet } & \mathrm{m}^{2} \\ b & \text { Inertial coefficient } & / \mathrm{m} \\ c & \text { Specific heat } & \mathrm{J} /(\mathrm{kgK}) \\ d_{m} & \text { Mean pore diameter } & \mathrm{m} \\ E_{S} & \text { Storage energy } & \mathrm{J} \\ h_{V} & \text { Volumetric heat transfer coefficient } & \mathrm{W} /\left(\mathrm{m}^{3} \mathrm{~K}\right) \\ K & \text { Permeability } & \mathrm{m}^{2} \\ k & \text { Thermal conductivity } & \mathrm{W} /(\mathrm{mK}) \\ L & \text { Chamber length } & \mathrm{m} \\ \mathcal{L} & \text { Value of shape parameter } & \\ P & \text { Average pressure } & \mathrm{Pa} \\ \mathcal{P} & \text { Shape parameter } & \mathrm{Pa} \\ p & \text { Local pressure } & \\ \mathrm{Pr} & \text { Prandtl number } & \mathrm{m} \\ R & \text { Radius } & \mathrm{m} \\ r & \text { Radial coordinate } & \\ R e & \text { Reynolds number } & \mathrm{Pa} / \mathrm{m} \\ S_{m} & \text { Momentum source term } & \mathrm{K} \\ T & \text { Local air temperature } & \mathrm{K} \\ T_{0} & \text { Initial temperature; wall temperature }\end{array}$




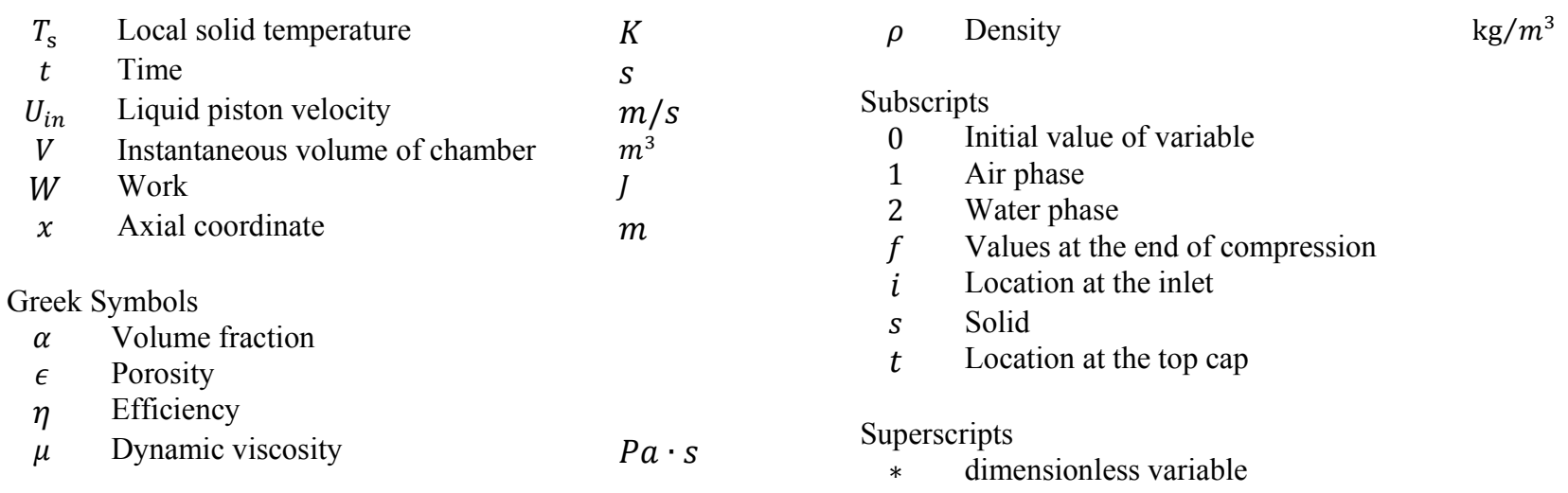

